\title{
Current Concepts in the Management of Herpes Simplex Anterior Segment Eye Disease
}

\author{
Jagadesh C. Reddy · Christopher J. Rapuano
}

Published online: 19 September 2013

(C) Springer Science + Business Media New York 2013

\begin{abstract}
Herpes simplex virus-1 (HSV-1) infects the majority of the world's population during their lifetime. The prevalence of HSV primarily depends on geographic location, socioeconomic status, and age. HSV ocular disease rarely manifests as primary disease and most commonly as a recurrent disease. Recurrent HSV affects all the anterior segment structures in various clinical forms. In the cornea, HSV manifests as epithelial, stromal and endothelial disease. Depending on the structure involved, signs, symptoms and management options differ. Early diagnosis and prompt and appropriate management can lead to early resolution of the disease and maintenance of anatomic and functional integrity of ocular structures.
\end{abstract}

Keywords Herpes simplex virus (HSV) - Blepharitis · Conjunctivitis - Keratitis · Latency · Dendritic ulcer · Geographic ulcer - Epithelial keratitis - Stromal keratitis · Necrotizing · Non-necrotizing $\cdot$ Immune-mediated · Endotheliitis · Iridocyclitis · Trabeculitis · Scleritis · Anti-viral · Keratoplasty

J. C. Reddy · C. J. Rapuano ( $\square)$

Cornea Service-Wills Eye Hospital, Jefferson Medical College of Thomas Jefferson University, 840 Walnut Street, Suite 920, Philadelphia, PA 19107, USA

e-mail: cjrapuano@willseye.org

J. C. Reddy

Cornea, Anterior Segment and Refractive Surgery Service, L V Prasad Eye Institute, Kallam Anji Reddy Campus, Banjara Hills Road No. 2, Hyderabad 500034, India

\section{Introduction}

Characteristic Features of Herpes Viruses

Human herpes viruses are ubiquitous agents distributed worldwide but restricted to humans as their natural host. Human infections are caused by nine distinct members of family herpesviridae. These virions consist of an icosahedral capsid surrounding the core, a largely unstructured tegument, an outer lipid bilayer envelope and a double-stranded DNA genome [1]

Herpes Simplex Virus (HSV) Diseases of the Anterior Segment and the Adnexa

Seroprevalence of HSV ranges from 50 to $90 \%$ in the general adult populations [2]. The annual incidence of all types of new ocular HSV infections has recently been estimated at 11.8 per 100,000 people in the US [3]. Primary ocular HSV-1 infections are rare and usually present as conjunctivitis, blepharoconjunctivitis, inflammatory vesicles and ulcers of the eyelids, and rarely include dendritic lesions in the corneal epithelium. Ocular infections most commonly result from reactivation of virus that originally established a latent infection in the trigeminal ganglion.

Ocular HSV disease mostly affects the anterior segment of the eye and its adnexa. Diseases of the posterior segment due to HSV are relatively rare. Our goal is to define current knowledge of the pathophysiology and clinical presentation patterns, diagnosis and management options of HSV-related disease of the anterior segment and identify areas of further research.

\section{Pathophysiology}

The pathophysiological characteristic feature of HSV is its ability to gain access to peripheral sensory neurons and 
attain latency in the nuclei of the associated neural ganglion cells. This attribute allows neuronal viral recurrences manifesting as various forms of peripheral herpetic disease. Nucleocapsids located in the ganglion are carried by anterograde axonal transport along microtubules to axon terminals where the egress of enveloped infectious particles takes place, allowing for trans-synaptic and further inter- cellular spread $[1,4]$.

The vast majority of primary infections pass subclinically or as such mild disease that a herpes etiology is not suspected. Primary ocular HSV disease is less common compared with labial herpes.

\section{Clinical Features and Management, Fig. 1}

\section{HSV Blepharitis}

Herpetic blepharitis can be a primary infection or recurrence. Clinical appearance is a vesicular lesion involving a focal area of the eyelid with surrounding erythema, which progresses to ulceration and crusting and heals without a scar. The disease can involve only the lids or can progress to keratitis. Diagnosis can be made by the typical clinical appearance and course of a recurrent, focal, vesicular lesion, and also through viral culture $[5,6]$.

\section{Current Management}

Observation, or a short course of topical acyclovir ointment $(3 \%)$ or ganciclovir gel $(0.15 \%)$ or vidarabine ointment ( $3 \%)$ to the eyelid skin or oral antiviral medications if the eyelid margin is not involved. If the eyelid margin is involved, a short course of topical antiviral medications [e.g., acyclovir ointment $(3 \%)$ or ganciclovir gel $(0.15 \%)$ or vidarabine ointment (3\%) 3-4 $\times$ days for 1-2 weeks] to the eyelid skin and eyes or oral antiviral medications (e.g., acyclovir $400 \mathrm{mg}$ 5 times a day, valacyclovir $500 \mathrm{mg} 3$ times a day or famciclovir $250 \mathrm{mg} 3$ times a day for 1-2 weeks) is used.

\section{HSV Conjunctivitis}

HSV conjunctivitis can present as recurrent follicular conjunctivitis. It is usually self-limited, but it may lead to a subsequent conjunctival dendritiform ulcer or dendritic keratitis. Conjunctival epithelial defects lack the classic tree branch features of corneal dendrites.

\section{Current Management}

A short course of topical antiviral or oral antiviral medications [e.g., acyclovir ointment (3\%) or ganciclovir gel $(0.15 \%)$ or vidarabine ointment $(3 \%) 3-4 \times$ days for
1-2 weeks] or oral antiviral medications (e.g., acyclovir $400 \mathrm{mg} 5$ times a day, valacyclovir $500 \mathrm{mg} 3$ times a day or famciclovir $250 \mathrm{mg} 3$ times a day for $1-2$ weeks).

\section{HSV Epithelial Keratitis}

Epithelial keratitis starts as punctate vesicular eruptions in the corneal epithelium, but they quickly coalesce into dendritic tree branch-shaped lesions. The branches are bordered by a reticulate band of punctate epithelial microdestruction, and the ends terminate in end-bulbs (swollen epithelial borders that contain live virus). The distinct pattern of the epithelial lesion is evident from the staining of the basement membrane with fluorescein dye and staining of the damaged cells at the edges with rose bengal dye. An enlarged dendritic ulcer leads to formation of a geographic ulcer. A geographic ulcer can be differentiated from healing abrasions and neurotrophic keratopathy by the presence of scalloped or serpiginous borders and a staining pattern as seen in a dendritic ulcer [7].

As epithelial cells lodge active virus, antiviral therapy should be initiated. Ophthalmic formulations of trifluridine $(1 \%)$, acyclovir $(3 \%)$ and ganciclovir $(0.15 \%)$ are effective and safe in the acute treatment of HSV epithelial keratitis $[8 \bullet \bullet, 9]$. Trifluridine, a fluorinated pyrimidine nucleoside, acts as a thymidine analog. It has better or equal efficacy compared to older topical agents [10]. Trifluridine is activated by viral and host cell thymidine kinase and is integrated into the DNA of both the herpes virus and host cells. Trifluridine should not be used for a prolonged duration because of its severe ocular toxicity. It has low solubility, and the corneal penetration is limited when the corneal epithelium is intact. Ganciclovir, a newer agent which is less toxic to the ocular surface and very effective in the treatment of HSV dendrites, has largely replaced trifluridine, which was once most commonly used. There are no studies directly comparing trifluridine and ganciclovir, but indirectly trifluridine has been shown to be comparable to acyclovir in the healing rates of HSV epithelial keratitis $[8 \cdot \bullet, 9]$.

Acyclovir is a synthetic purine nucleoside analog that is converted to acyclovir monophosphate by the virus-encoded enzyme thymidine kinase. Acyclovir monophosphate is converted to acyclovir triphosphate by human enzymes. Acyclovir triphosphate selectively inhibits viral DNA polymerase. Acyclovir ophthalmic ointment is currently available in Europe and Asia. Acyclovir $3 \%$ eye ointment exhibited greater or equal effectiveness compared to other topical antiviral medications tested. Patients treated with acyclovir eye ointment healed faster compared to those treated with topical idoxuridine and adenine arabinoside, but there was no statistical difference in healing time when compared with trifluridine and vidarabine [8••, 11-14]. 

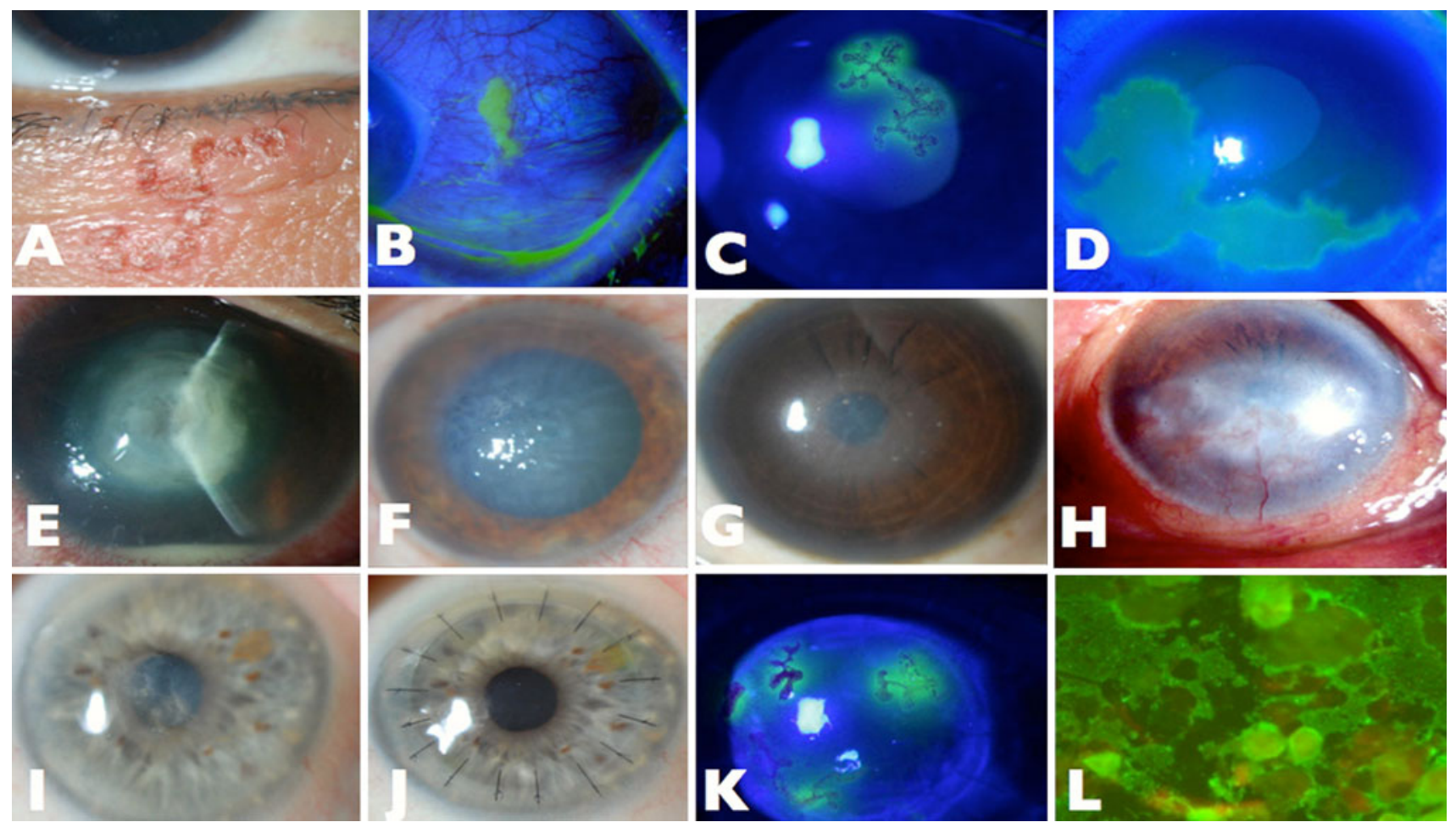

Fig. 1 Clinical photographs (a). Right eye of a patient with herpetic blepharitis showing ulcerated and crusted lesions on the lower lid (b). Left eye of a patient with conjunctival congestion and dendritiform ulcer stained with fluorescein stain (c). Corneal epithelial dendrite with the base stained with fluorescein and the edges with rose bengal (d). Geographic ulcer stained with fluorescein (e). Left eye of a patient with infiltrate, necrosis, thinning, descemetocele, impending perforation and hypopyon: necrotizing stromal keratitis (f). Left eye of a patient with central corneal stromal edema with peripheral clear cornea and no keratic precipitates (KPs: non-necrotizing stromal

Ganciclovir is a derivative of acycloguanosine, which is transformed into triphosphate ganciclovir in vivo. Triphosphate deoxyguanosine embeds into the virus deoxyribonucleic acid (DNA) via competitive inhibition and consequently inhibits the DNA polymerase of the herpes simplex virus, slows the replication of virus DNA chains and inhibits the synthesis of virus DNA $[15,16]$.

Studies have shown better or equal efficacy of ganciclovir compared to acyclovir eye ointment. Topical ganciclovir ophthalmic gel has good tolerance and prolonged corneal contact time. It is as effective as acyclovir but at a much lower concentration. Ganciclovir ophthalmic gel has been approved for treatment of acute HSV epithelial ker-

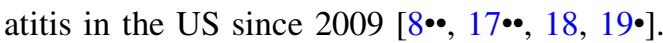

Interferons are cytokines capable of activating an intracellular pathway that upregulates host genes affecting antiviral responses. Interferon is shown to have antiviral activity against HSV-infected epithelial cells. Studies of combined interferon and antiviral agents had inconsistent results, probably because different concentrations of keratitis, g. Central corneal edema with underlying keratic precipitates and surrounding clear cornea: disciform endotheliitis (h). Right eye of a patient with a vascularized corneal scar that developed after resolution of necrotizing stromal keratitis (i). Cornea with a lamellar corneal scar developed after resolution of necrotizing stromal keratitis that was treated with lamellar keratoplasty $(\mathbf{j}, \mathbf{k})$. Recurrence of HSV epithelial keratitis in a 7-year-old graft corneal graft done for a vascularized corneal scar (I). Immunofluorescence assay slide showing cells exhibiting bright apple-green fluorescence, which suggests positivity for HSV-1 (Color figure online)

interferon were used. Higher interferon concentrations were generally more effective than lower concentrations.

The combination of interferon and an antiviral agent was not significantly better than antiviral monotherapy at 14 days but provided significantly faster healing [20, 21].

There was no statistically significant difference in the healing time in patients treated with oral or topical acyclovir [22]. Oral acyclovir is especially useful for treating infants and children with infectious epithelial keratitis because it is difficult to administer topical medications in this age group, and infants and children tend to express a severe immune reaction associated with their epithelial disease [23].

Topical antiviral therapy should be continued for 10-14 days. If there is no resolution of the epithelial keratitis in 2 weeks, the diagnosis of HSV epithelial keratitis should be reconsidered and the eye carefully examined so as not to miss an infectious keratitis due to other organisms (e.g., acanthamoeba or slow-growing bacterial infections such as atypical mycobacteria and Nocardia; fungus is less likely) or a neurotrophic keratopathy. If the lesion is consistent with 
persistent HSV epithelial keratitis, resistance to the antiviral medication, while rare, must be considered, and an alternative medication can be initiated [24].

Physical debridement of the ulcer with a sterile cottontipped applicator was widely practiced in the past, but its role in HSV epithelial keratitis is questionable because of the risk of damaging Bowman's layer and the potential of increased corneal inflammation or scarring and continued shedding of the virus for several days after debridement. Some physicians will debride corneal dendrites with the goal of eliminating a significant viral load, but will also use anti-viral medications after the debridement.

Topical cycloplegics may be beneficial in patients with significant photophobia or ciliary spasm. Topical corticosteroid medications are not recommended for the treatment of infectious epithelial keratitis. If severe immune stromal inflammation is also present, topical steroids can be used very judiciously, but ideally after antiviral medications have been started and the active infection is improving.

\section{Herpetic Eye Disease Study: The Epithelial Keratitis Trial [25]}

In this study, the efficacy of oral acyclovir for the prevention of stromal keratitis or iritis in patients with HSV epithelial keratitis was evaluated. These patients were randomly assigned to receive a 3 -week course of oral acyclovir, $400 \mathrm{mg} 5$ times a day, or placebo in addition to topical trifluridine for their epithelial disease. Patients were assessed during a 12-month follow-up period. The study concluded that for patients with HSV epithelial keratitis treated with topical trifluridine, there was no apparent benefit of a 3-week course of oral acyclovir in preventing HSV stromal keratitis or iritis.

\section{Current Management}

A short course of a topical antiviral (e.g., trifluridine 9 times a day for 1 week, then $5 \times$ days for 1 week or ganciclovir 5 times a day for 1 week, then $3 \times$ days for 1 week) or oral antiviral medications (e.g., acyclovir $400 \mathrm{mg} 5$ times a day, valacyclovir $500 \mathrm{mg} 3$ times a day or famciclovir $250 \mathrm{mg} 3$ times a day for 2 weeks).

\section{HSV Stromal Keratitis}

Stromal disease accounts for approximately $2 \%$ of initial episodes of ocular HSV disease, but it accounts for 20-48 \% of recurrent ocular HSV disease [26-28]. Two manifestations of stromal disease from HSV involve the stroma primarily. Necrotizing stromal keratitis occurs from active infection, whereas immune stromal keratitis is an immune reaction.
Necrotizing stromal keratitis is a manifestation of direct viral invasion of the corneal stroma. Active viral replication as well as related immune-mediated tissue damage contributes to corneal injury. The clinical findings are necrosis, ulceration and dense infiltration of the stroma with an overlying epithelial defect. Severe infection/inflammation may lead to thinning and perforation. Electron microscopic examination of pathological specimens from patients with necrotizing stromal keratitis has demonstrated virions in stromal keratocytes [29, 30••, $31 \bullet \bullet$.

Non-necrotizing keratitis, also referred to as immune stromal keratitis, is much more common than necrotizing keratitis and involves stromal inflammation without an associated epithelial defect. Stromal infiltration is often accompanied by corneal edema, anterior chamber inflammation and ciliary flush [32]. The role of retained viral antigen as a stimulus for chronic inflammation (focal, multifocal or diffuse) and $\mathrm{T}$ cell-mediated autoimmune responses in murine models have been studied but the role of live virus in the pathogenesis of immune stromal keratitis has not been elucidated fully [33]. It is hypothesized that stromal inflammation is driven by either HSV-1-specific CD4p $\mathrm{T}$ cells stimulated directly by viral antigens, bystander cytokine activation of CD4p T cells, auto-antigens unmasked and mimicked by HSV-1 corneal infection or a combination of these processes [34].

Stromal keratitis is often a chronic, recurrent inflammation with fluctuations in severity that require long-term topical corticosteroids. Untreated or undertreated inflammation can lead to stromal scarring, thinning, persistent neovascularization, lipid deposition and severe loss of vision [35].

Topical corticosteroids and antivirals are the current mainstay of therapy for HSV stromal keratitis. Corticosteroids are used to combat the immunological component of stromal disease and the antivirals to inactivate and prevent further viral replication.

\section{Herpetic Eye Disease Study}

Multicenter, randomized, placebo-controlled trials were performed to answer the following questions for the management of stromal keratitis:

A. Topical Corticosteroids in Treating Stromal Keratitis in Patients Already on a Topical Antiviral Medication [36•] In this study, the efficacy of topical corticosteroids in the treatment of HSV stromal keratitis was evaluated. Patients with active HSV stromal keratitis who had not received any corticosteroids for at least 10 days before recruitment were enrolled. These patients were categorized into the placebo or steroid group. Both regimens were 
tapered over 10 weeks, and both groups received prophylactic topical trifluridine.

Patients treated with topical steroids demonstrated less persistence or progression of stromal inflammation compared to the placebo group in addition to a shorter duration of disease. There was no effect on the visual acuity at 6 months in delaying the steroid treatment for a few weeks. The incidence of recurrent herpes was not affected by topical steroid use.

\section{B. Oral Acyclovir in Treating Stromal Keratitis in Patients} Already on a Topical Steroid and Antiviral Medication [37.] In this study, the efficacy of oral acyclovir in treating HSV stromal keratitis was evaluated. These patients were randomized to receive a 10-week course of either oral acyclovir (400 mg 5 times daily) or placebo. All patients also received a standard regimen of topical prednisone phosphate and trifluridine.

The study showed that there was no clinically significant beneficial effect of oral acyclovir in treating HSV stromal keratitis in patients receiving topical corticosteroids and trifluridine. Visual acuity, however, improved over 6 months in significantly more patients in the acyclovir group than in the placebo group.

\section{Current Management}

Necrotizing keratitis Topical antibiotics, very judicious use of topical steroids and the treatment dose of oral antiviral medications (e.g., acyclovir $400 \mathrm{mg} 5$ times a day, valacyclovir $500 \mathrm{mg} 3$ times a day or famciclovir $250 \mathrm{mg} 3$ times a day for 1-2 weeks).

Immune stromal keratitis Observation if mild. Topical steroids with topical antiviral medications or, more commonly, a prophylactic dose of oral antiviral medications (e.g., acyclovir $400 \mathrm{mg} 2$ times a day, valacyclovir $500 \mathrm{mg}$ 1-2 times a day or famciclovir $250 \mathrm{mg} \mathrm{1-2} \mathrm{times} \mathrm{a} \mathrm{day).}$ When used, the topical steroids typically need to be tapered slowly and continued for weeks to months and occasionally for many years.

In eyes with a corneal perforation, corneal gluing should be considered if the perforation is small. In cases of large perforations, a lamellar patch graft or penetrating keratoplasty is typically required for tectonic support.

\section{HSV Endotheliitis}

HSV endotheliitis manifests as corneal edema (epithelial and stromal) without corneal infiltrate with underlying keratic precipitates and iritis. Inflammation of the endothelium leads to iritis and corneal edema. The cornea beyond the area of KPs is clear and uninvolved. The exact pathogenesis of endotheliitis in HSV is unknown.
Immunological and live viruses as etiological agents have been speculated, but response to steroids supports an immunological hypothesis [38, 39]. Based on the configuration of the stromal edema and the distribution of KPs, HSV endotheliitis is further classified into disciform, diffuse and linear endotheliitis.

\section{Disciform Endotheliitis}

This is the most common form and is characterized by a round area of stromal edema with underlying KPs. Patients usually present with photophobia, decreased visual acuity and ocular discomfort. Limbal injection and a round area of stromal edema (disc shaped) overlying multiple KPs is usually seen. If untreated, disciform keratitis can lead to intractable edema.

The term disciform keratitis is often used interchangeably with immune-mediated stromal keratitis. This is because the shape of the edema is similar in both the entities. The clinician should be aware that immune stromal keratitis is due to direct stromal inflammation and may be associated with infiltrate or vascularization with no KPs, whereas disciform endotheliitis is the secondary stromal edema overlying KPs with no infiltrate or vascularization. This distinction between stromal and endothelial inflammation may be important, but the treatment and outcomes are similar.

\section{Diffuse Endotheliitis}

Patients present with pain, photophobia, injection and decreased vision. Clinically, these patients have diffuse corneal edema and scattered KPs; rarely, retrocorneal plaque of inflammatory cells or the hypopyon may be seen in severe cases. If not treated, it may lead to scarring, neovascularization, persistent edema and loss of vision.

Disciform as well as diffuse endotheliitis is normally exquisitely sensitive to topical corticosteroids, and early intervention usually leads to complete resolution of the edema and KPs without stromal scarring or loss of vision.

\section{Linear Endotheliitis}

Clinically, a line of KP is seen on the corneal endothelium that progresses centrally from the limbus. A well-demarcated line between the area of edematous and non-edematous cornea with the KP located at the leading edge of the edema is seen. Failure to recognize and properly treat this condition leads to corneal decompensation. Linear endotheliitis is quite difficult to treat. These patients should be treated aggressively with both corticosteroids and antiviral agents. Oral anti-viral medications may have a beneficial role and should be considered in all cases [40, 41]. 
Current Management Topical steroids with treatment dose of oral antiviral medications (e.g., acyclovir $400 \mathrm{mg} 5$ times a day, valacyclovir $500 \mathrm{mg} 3$ times a day or famciclovir $250 \mathrm{mg} 3$ times a day).

HSV Iridocyclitis, Trabeculitis and Acute Secondary Glaucoma

HSV iridocyclitis can occur with or without a prior history of keratitis. Patients present with photophobia, pain and ciliary flush. KPs and anterior chamber cellular reaction as well as segmental iris atrophy due to ischemic necrosis of the iris stroma are usually seen.

While most forms of iridocyclitis are associated with reduced intraocular pressure, HSV trabeculitis often results in acute severe elevation of intraocular pressure, which is due to blockage of aqueous outflow by inflammatory cells or primary trabeculitis. Consequently, when elevated intraocular pressure is found in association with iridocyclitis, a herpetic (herpes simplex or herpes zoster) etiology should be suspected. The pressure rise in these cases typically responds quickly to topical corticosteroids.

Pathogenesis of HSV iridocyclitis is not fully understood, but HSV particles were seen in iris tissue, and live viruses were isolated from anterior chamber of patients with HSV iridocyclitis [38, 42].

\section{Herpetic Eye Disease Study: A Controlled Trial of Oral Acyclovir for Iridocyclitis Caused by Herpes Simplex Virus}

This study was designed to evaluate the benefit of oral acyclovir for the treatment of HSV iridocyclitis already on topical prednisolone phosphate and trifluridine. Patients were randomly assigned to receive a 10-week course of either acyclovir $400 \mathrm{mg}$, 5 times daily, or oral placebo. The study was terminated early because of slow recruitment.

Treatment failure (persistence or worsening of ocular inflammation, withdrawal of medication because of toxicity or request by the patient to withdraw from the trial for any reason) occurred in $50 \%$ of the 22 patients in the acyclovir-treated group and in $68 \%$ of the 28 patients in the placebo group. The possible benefit of acyclovir became apparent after 3 weeks of follow-up. The results suggested a benefit of oral acyclovir in the treatment of HSV iridocyclitis, but the number of patients studied was too small to achieve statistically conclusive results [43].

\section{Current Management}

Topical steroids with a treatment dose of oral antiviral medications (e.g., acyclovir $400 \mathrm{mg} 5$ times a day, valacyclovir $500 \mathrm{mg} 3$ times a day or famciclovir $250 \mathrm{mg} 3$ times a day for 1-2 weeks). Depending on the intraocular pressure and the status of the optic nerve, pressure-lowering medications may also be required.

\section{HSV Scleritis}

Herpetic scleritis accounts for about $7 \%$ of all scleritis. The diagnosis is usually challenging and should be suspected if the presentation is a unilateral, sudden-onset, anterior scleritis associated with moderate to intense pain. These eyes can also have associated keratitis and anterior uveitis. It is usually suspected when scleritis is not responsive to steroid treatment.

Diagnosis is usually clinical, but scleral biopsy can be very helpful. In suspicious cases, a response to treatment trial of oral acyclovir (400 mg 5 times daily) might be considered. The response to oral acyclovir is usually dramatic and resolves the symptoms rapidly (within 3 weeks) in patients with herpes simplex scleritis. There is no consensus regarding how long this treatment should be continued, but clinical signs and symptoms may aid in discontinuing the treatment [44••].

\section{Current management}

Treatment dose of oral antiviral medications (e.g., acyclovir $400 \mathrm{mg} 5$ times a day, valacyclovir $500 \mathrm{mg} 3$ times a day or famciclovir $250 \mathrm{mg} 3$ times a day for 1-2 weeks). Judicious use of topical steroids may be helpful.

\section{Neurotrophic Keratopathy}

Neurotrophic keratopathy develops after non-healing or improper treatment of infectious epithelial keratitis. It is due to impaired corneal innervation in combination with decreased tear secretion, continuing inflammation and toxicity of topical medications (antivirals and steroids). It initially presents as loss of normal corneal luster, irregularity of the corneal surface leading to punctate epithelial erosions progressing to a persistent epithelial defect. The epithelial defect is most commonly located in interpalpebral area and is oval in shape with smooth, rounded borders. Persistence of the epithelial defect leads to stromal ulceration, stromal scarring, neovascularization, secondary bacterial infection and perforation.

It is usually managed by discontinuation of all unnecessary topical medications and frequent use of non-preservative artificial gels or ointments. In non-responsive cases, gentle debridement of abnormal epithelium at the ulcer edge may be beneficial. In recalcitrant cases a temporary tarsorrhaphy may be performed by injecting botulinum toxin into the levator muscle or suture tarsorrhaphy may be required. Covering the involved area with an amniotic membrane, either as a biologic bandage secured 
to a plastic ring or under a contact lens, or sutured or glued to the eye, can also be very helpful in some cases.

\section{Current Management}

Discontinue toxic topical medications. Frequent preservative-free lubrication, tarsorrhaphy and amniotic membrane coverage. Prophylactic dose of oral antiviral medications may be helpful (e.g., acyclovir $400 \mathrm{mg} 2$ times a day, valacyclovir $500 \mathrm{mg} \mathrm{1-2} \mathrm{times} \mathrm{a} \mathrm{day} \mathrm{or} \mathrm{famciclovir}$ $250 \mathrm{mg} 1-2$ times a day).

\section{HSV Scarring}

Due to infiltrate per se or chronic inflammation, corneal scarring and vascularization can develop. If there is significant corneal irregularity and only mild scarring, a rigid gas-permeable contact lens may greatly improve the vision. In order to visually rehabilitate eyes with moderate to severe scarring, corneal transplantation often needs to be performed. The literature has shown that there is a higher than average risk of graft failure in patients who undergo penetrating corneal transplantation for scars due to herpes simplex keratitis. The causes of failure are multifactorial and incompletely understood, but often involve recurrent HSV infection/inflammation or sequelae of neurotrophic keratopathy. There is a higher incidence of immunologic rejection caused by HSV-specific factors (persistence of inflammation, vascularization), and recurrence of HSV disease compromises graft survival. Graft rejection or its treatment could themselves induce reactivation or provoke recurrent disease by creating more favorable conditions for virus replication [45-47].

In order to decrease the graft failure rate due to endothelial rejection and decompensation, anterior lamellar keratoplasty has been adopted as a treatment for many of these eyes with a good outcome. Deep anterior lamellar keratoplasty is an alternative and safe procedure to restore vision in cases with significant corneal scarring due to recurrent HSV keratitis with healthy endothelium. Sarnicola and Toro have shown excellent success with anterior lamellar keratoplasty with no evidence of recurrence and rejection over a follow-up period of 31 months [48••]. Additionally, it has been demonstrated that postoperative antiviral prophylaxis reduces the rate of recurrence of HSV disease and also rejection episodes and thus graft failure [49].

\section{Herpetic Eye Disease Study: Acyclovir for the Prevention of Recurrent Herpes Simplex Virus Eye Disease [50••]}

This study was designed to evaluate the efficacy of oral acyclovir as a prophylaxis for the prevention of recurrent ocular HSV disease. Seven hundred three patients with a history of ocular HSV disease within the preceding year were randomly assigned to receive $400 \mathrm{mg}$ of acyclovir $(n=357)$ or placebo $(n=346)$ orally twice daily. The study outcomes were the rates of development of ocular or nonocular HSV disease during a 12-month treatment period and a subsequent 6-month observation period.

The study concluded that long-term oral prophylaxis is effective in reducing the rate of recurrent ocular HSV disease and orofacial HSV disease, and is most important for the subset of patients with a previous history of HSV stromal keratitis.

\section{Current Management}

Chronic low-dose topical steroids may be required to prevent episodes of active inflammation. Most patients are treated with a chronic prophylactic dose of oral antiviral medications (e.g., acyclovir $400 \mathrm{mg} 2$ times a day, valacyclovir $500 \mathrm{mg} 1-2$ times a day or famciclovir $250 \mathrm{mg}$ 1-2 times a day). Depending on the degree of visual loss and scarring, a contact lens, lamellar keratoplasty or penetrating keratoplasty may be beneficial. Chronic prophylactic oral antiviral medications are used after corneal transplanation for eyes with HSV keratitis.

In recent years there has been increased interest in resistance to acyclovir due to long-term prophylactic usage. Resistance to acyclovir is rare in immunocompetent hosts $(\leq 1 \%)$ but is more commonly seen in immunocompromised hosts (4-7\%) [51]. The use of limited ACV prophylaxis, e.g., stopping prophylactic treatment after 1 year and then reinstating therapeutic treatment for subsequent recurrence or perhaps a combination antiviral therapy that includes new drugs with distinct mechanisms of action may be required in the future in order to prevent the emergence of resistant HSV strains [52].

\section{Diagnostic Modalities: Laboratory and Imaging}

Isolation of herpes simplex virus-1 (HSV-1) in culture provides the most reliable and specific method, and is considered the gold standard in laboratory diagnosis of herpes simplex keratitis. Other rapid diagnostic tests for HSV include Giemsa staining, enzyme- or fluorescencebased immunological detection of HSV-1 antigen and polymerase chain reaction-based detection of viral DNA.

The smear for Giemsa stain is stained for the observation of multinucleated giant cells (MNGCs) and intranuclear inclusions. It has a sensitivity of up to $57 \%$ and specificity of $86 \%$ [53]. 
Immunofluorescence Assay (IFA)

It is said to be positive for HSV-1 if the cells exhibit specific bright apple-green fluorescence. A minimum of 20 basal epithelial cells were examined before the smear was considered negative. It has a sensitivity of up to $86 \%$ and specificity of $85 \%$. Polymerase chain reaction (PCR) has a $100 \%$ sensitive and $68 \%$ specific in the diagnosis of HSV keratitis [53].

In view of virus culture having a very low positivity in corneal scraping and the need for a well-equipped virology laboratory, a combination of PCR and IFA tests is optimum to achieve a reliable diagnosis of HSV keratitis, and the presence of MNGCs in routine Giemsa-stained corneal scrapings may provide a useful clue in unsuspected cases [53, 54].

Other diagnostic tools include electron microscopy (directly view herpes virions) and DNA hybridization techniques using HSV nucleic acid probes. The technical difficulties associated with these methods preclude widespread clinical us in spite of these tests being highly sensitive and specific.

Imaging technologies such as in vivo confocal microscopy can be used for characterization of corneal inflammation and also used for monitoring inflammation. It has been shown that the inflammatory response is characterized by cellular infiltration of all corneal layers, attenuation of the subbasal nerve plexus and endothelial cell loss [55].

\section{Recent Developments: Newer Drug Formulations and Vaccination}

Newer oral antivirals have been made available that simplify the dosing regimen of acyclovir. Valacyclovir and famciclovir act through inhibition of viral DNA polymerase, like acyclovir. Although their efficacy in humans is to be ascertained, convenient twice-a-day dosage (once for prophylaxis) has led to their widespread use, despite higher cost.

The use of topical cyclosporine A has been attempted with variable success rates in the management of HSV stromal keratitis. These studies contained small numbers of herpetic eyes, and all lacked a control group [56, 57].

In a mouse model, vaccination with a mixture of selfadjuvanting lipopeptides containing novel HSV-1 immunodominant $\mathrm{gD}$ T-cell epitopes increased protection from ocular herpes infection and disease. But the strength of this protective immunity induced by these lipopeptides together with their safety need to be ascertained to combat ocular herpes infection and disease in humans [58].

\section{Scope for Research}

In HSV epithelial keratitis, corneal re-epithelialization is a key measure of the therapeutic efficacy of treatment modalities, but ancillary outcomes including residual corneal opacification, visual acuity and quality of life should also be taken into consideration.

In this new era of gene therapy, the potential treatment of many genetic diseases and viral infections with gene modification is worthy of attention in the management of HSV ocular disease. Therapeutic genes or molecules can be transferred to the cornea by direct application of naked DNA, ballistic transfer with a gene gun, viral and non-viral vectors. Research regarding the role of direct gene transfer on chronic inflamed cornea of HSV disease or on eye bank donor corneas before transplantation to prevent recurrence and failure could be potential targets.

\section{Conclusion}

In the present scenario, there are still many questions to be answered about the pathogenesis, recurrences and medical treatment of ocular herpes simplex. The HEDS was limited to the use of oral acyclovir and topical trifluridine; with the advent of newer drug formulations, further research is warranted to prove the beneficial effect of these drugs. Many humans harbor latent HSV-1 in their trigeminal ganglia; recurrences can be prevented by eliminating the virus in its latent stage in the trigeminal ganglion and other ganglia around the eye. Advances in immunology and molecular virology may aid in eradicating herpes keratitis.

Acknowledgments No author has a financial or proprietary interest in any material or method mentioned.

Compliance with Ethics Guidelines This article does not contain any studies with human or animal subjects performed by any of the authors.

Disclosure Jagadesh Reddy declares that he has no conflict of interest. Christopher Rapuano has been a consultant to Allergan, Bausch \& Lomb, Bio-Tissue and Merck and has stock/stock options with Rapid Pathogen Systems.

\section{References}

Papers of particular interest, published recently, have been highlighted as:

- Of importance

•• Of major importance

1. Hartmut Hengel. Common characteristics and distinct features of human pathogenic herpesviruses. In: Rainer Sundmacher, editor. Color atlas of herpetic eye diseases-a practical guide to clinical management. Heidelberg: Springer; 2009. p. 1-4.

2. Rabenau HF, Buxbaum S, Preiser W, Weber B, Doerr HW. Seroprevalence of herpes simplex virus types 1 and type 2 in the 
Frankfurt am Main area, Germany. Med Microbiol Immunol. 2002;190:153-60.

3. Young RC, Hodge DO, Liesegang TJ, Baratz KH. Incidence, recurrence, and outcomes of herpes simplex virus eye disease in Olmsted County, Minnesota, 1976-2007: the effect of oral antiviral prophylaxis. Arch Ophthalmol. 2010;128:1178-83.

4. Tullo A. Pathogenesis and management of herpes simplex virus keratitis. Eye (Lond). 2003;17:919-22.

5. Liu S, Pavan-Langston D, Colby KA. Pediatric herpes simplex of the anterior segment: characteristics, treatment, and outcomes. Ophthalmology. 2012;119:2003-8.

6. Revere K, Davidson SL. Update on management of herpes keratitis in children. Curr Opin Ophthalmol. 2013;24:343-7.

7. Wilhelmus KR, et al. Prognostic indicators of herpetic keratitis: analysis of a five-year observation period after corneal ulceration. Arch Ophthalmol. 1981;99:1578-82.

8. $\bullet$ Wilhelmus KR. Antiviral treatment and other therapeutic interventions for herpes simplex virus epithelial keratitis. Cochrane Database Syst Rev. 2010;8:CD002898. doi: 10.1002/ 14651858.CD002898. This comprehensive review of the world literature compared the relative effectiveness of antiviral agents, interferon, and corneal débridement in the treatment of acute HSV epithelial keratitis.

9. Kaufman HE, Haw WH. Ganciclovir ophthalmic gel $0.15 \%$ : safety and efficacy of a new treatment for herpes simplex keratitis. Curr Eye Res. 2012;37:654-60.

10. Power WJ. Benedict-Smith a, hillery $M$, et al. Randomised double blind trial of bromovinyldeoxyuridine (BVDu) and trifluoro- thymidine (Tf) in dendritic corneal ulceration. Br J Ophthalmol. 1991;75:649-51.

11. Collum LM, Benedict-Smith A, Hillary IB. Randomised doubleblind trial of acyclovir and idoxuridine in dendritic corneal ulceration. Br J Ophthalmol. 1980;64:766-9.

12. Young BJ, Patterson A, Ravenscroft T. A randomised double-blind clinical trial of acyclovir (Zovirax) and adenine arabinoside in herpes simplex corneal ulceration. Br J Ophthalmol. 1982;66:361-3.

13. Hovding G. A comparison between acyclovir and trifluorothymidine ophthalmic ointment in the treatment of epithelial dendritic keratitis. a double blind, randomized parallel group trial. Acta Ophthalmol. 1989;67:51-4.

14. Laibson PR, Pavan-Langston D, Yeakley WR, Lass J. Acyclovir and vidarabine for the treatment of herpes simplex keratitis. Am J Med. 1982;73:281-5.

15. Shiota H, Naito T, Mimura Y. Anti-herpes simplex virus (HSV) effect of 9-(1,3-dihydroxy-2-propoxymethyl)guanine (DHPG) in rabbit cornea. Curr Eye Res. 1987;6:241-5.

16. Croxtall JD. Ganciclovir ophthalmic gel $0.15 \%$ : in acute herpetic keratitis (dendritic ulcers). Drugs. 2011;26(71):603-10.

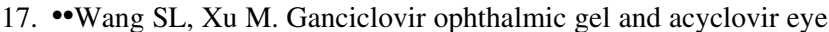
drops: comparative efficacy in treatment of viral keratitis. China Mod Med. 2009;16:53. This study compares ganciclovir gels in different concentrations with a placebo gel and $3 \%$ acyclovir ophthalmic ointment in the treatment of HSV-1 rabbit keratitis.

18. Colin J. Ganciclovir ophthalmic gel, $0.15 \%$ : a valuable tool for treating ocular herpes. Clin Ophthalmol. 2007;1:441-53.

19. - Sahin A, Hamrah P. Acute herpetic keratitis: what is the role for ganciclovir ophthalmic gel?. Ophthalmol Eye Dis. 2012;19:23-34. This paper reviews the pharmacology, efficacy, side effects and role of ganciclovir ophthalmic gel $0.15 \%$ in the treatment of acute herpetic keratitis.

20. Colin J, Chastel C, Renard G, Cantell K. Combination therapy for dendritic keratitis with human leukocyte interferon and acyclovir. Am J Ophthalmol. 1983;95:346-8.

21. De Koning EW, van Bijsterveld OP, Cantell K. Combination therapy for dendritic keratitis with human leukocyte interferon and trifluorothymidine. Br J Ophthalmol. 1982;66:509-12.
22. Collum 1M, McGettrick P, Akhtar J, et al. Oral acyclovir (Zovirax) in herpes simplex dendritic corneal ulceration. Br J Ophthalmol. 1986;70:435-8.

23. Schwartz GS, Holland EJ. Oral acyclovir for the management of herpes simplex virus keratitis in children. Ophthalmology. 2000;107:278-82.

24. Zhang W, et al. Dendritic keratitis caused by an acyclovirresistant herpes simplex virus with frameshift mutation. Cornea. 2007;26:105-6.

25. Herpetic Eye Disease Study Group. The epithelial keratitis trial. A controlled trial of oral acyclovir for the prevention of stromal keratitis or iritis in patients with herpes simplex virus epithelial keratitis. Arch Ophthalmol. 1997;115:703-12.

26. Liesegang TJ. Epidemiology of ocular herpes simplex. Arch Ophthalmol. 1989;107:1160-5.

27. Whitcher JP, et al. Herpes simplex keratitis in a developing country. Arch Ophthalmol. 1976;94:587-92.

28. Farooq AV, Shukla D. Herpes simplex epithelial and stromal keratitis: an epidemiologic update. Surv Ophthalmol. 2012;57:448-62.

29. Brik D, Dunkel E, Pavan-Langston D. Persistent herpes simplex virus in the corneal stroma despite topical and systemic antiviral therapy. Arch Ophthalmol. 1993;111:522.

30. • Rowe AM, St Leger AJ, Jeon S, et al. Herpes keratitis. Prog Retin Eye Res. 2013;32:88-101. This article gives an excellent overview of our knowledge of the pathogenic mechanisms and recurrent nature of HSK.

31. • Edward J. Holland, Gary S. Schwartz, Kristiana D. Neff. Herpes Simplex Keratitis. Cornea- Fundamentals, diagnosis and Managament. In: Jay H Krachmer, Mark J Mannis, Edward J Holland, editors. Chapter 79. This chapter describes in detail the clinical presentation patterns and management of all the HSV anterior segment diseases. 3rd ed. Amsterdam: Elsevier; 2011.

32. Wilhelmus KR. Diagnosis and management of herpes simplex stromal keratitis. Cornea. 1987;6:286-91.

33. Zhao ZS, et al. Molecular mimicry by herpes simplex virus-type 1: autoimmune disease after viral infection. Science. 1998;279: 1344-7.

34. Lepisto AJ, Frank GM, Hendricks RL. How herpes simplex virus type 1 rescinds corneal privilege. Chem Immunol Allergy. 2007; 92:203-12.

35. Giménez F, Suryawanshi A, Rouse BT. Pathogenesis of herpes stromal keratitis-a focus on corneal neovascularization. Prog Retin Eye Res. 2013;33:1-9.

36. - Wilhelmus KR, et al. Herpetic Eye Disease Study. A controlled trial of topical corticosteroids for herpes simplex stromal keratitis. Ophthalmology. 1994;101:1883-1895. (discussion 1994: 1895-1896). This study demonstrated that topical steroid treatment was significantly better than placebo in reducing persistence or progression of stromal inflammation and in shortening the duration of $H S V$ stromal keratitis.

37. - Barron BA, et al. Herpetic Eye Disease Study. A controlled trial of oral acyclovir for herpes simplex stromal keratitis. Ophthalmology. 1994;101:1871-1882. This study showed that there was no clinically significant beneficial effect of oral acyclovir in treating HSV stromal keratitis in patients receiving concomitant topical corticosteroids and trifluridine.

38. Kaufman HE, Kanai A, Ellison ED. Herpetic iritis: demonstration of virus in the anterior chamber by fluorescent antibody techniques and electron microscopy. Am J Ophthalmol. 1971;71:465.

39. Holbach L, Font R, Naumann G. Herpes simplex stromal and endothelial keratitis. Ophthalmology. 1990;97:722.

40. Fuchs A. Keratitis linearis migrans. Z Augenheilkd. 1926;58:315.

41. Khodadoust AA, Attarzadeh A. Presumed autoimmune corneal endotheliopathy. Am J Ophthalmol. 1982;93:718.

42. Witmer R, Iwamoto T. Electron microscope observation of herpes-like particles in the iris. Arch Ophthalmol. 1968;79:331. 
43. The Herpetic Eye Disease Study Group. A controlled trial of oral acyclovir for iridocyclitis caused by herpes simplex virus. Arch Ophthalmol. 1996;114:1065-72.

44. •- Gonzalez-Gonzalez LA, Molina-Prat N, Doctor P, et al. Clinical features and presentation of infectious scleritis from herpes viruses: a report of 35 cases. Ophthalmology. 2012;119:1460-1464. This study describes clinical features of herpetic scleritis and also highlights the clinical differences between patients with idiopathic scleritis and those with HSV.

45. Epstein RJ, Seedor JA, Dreizen NG, et al. Penetrating keratoplasty for herpes simplex keratitis and keratoconus: Allograft rejection and survival. Ophthalmology. 1987;94:935-44.

46. Ficker LA, Kirkness CM, Rice NSC, et al. Longterm prognosis for corneal grafting in herpes simplex keratitis. Eye. 1988;2: $400-8$.

47. Nicholls SM, Shimeld C, Easty DL, Hill TJ. Recurrent herpes simplex after corneal transplantation in rats. Invest Ophthalmol Vis Sci. 1996;37:425-35.

48. • Sarnicola V, Toro P. Deep anterior lamellar keratoplasty in herpes simplex corneal opacities. Cornea. 2010;29:60-64. This study shows that deep anterior lamellar keratoplasty is a safe procedure to restore vision in cases with significant corneal scarring due to recurrent HSV keratitis with healthy endothelium.

49. Garcia DD, Farjo Q, Musch DC, Sugar A. Effect of prophylactic oral acyclovir after penetrating keratoplasty for herpes simplex keratitis. Cornea. 2007;26:930-4.

50. • The Herpetic Eye Disease Study Group. Acyclovir for the prevention of recurrent herpes simplex virus eye disease. N Engl J Med. 1998;339:300-306. This paper describes the use of oral acyclovir after active herpetic ocular disease to prevent recurrences of ocular and nonocular herpes for 1 year during oral acyclovir treatment.

51. Bacon TH, Levin MJ, Leary JJ, Sarisky RT, Sutton D. Herpes simplex virus resistance to acyclovir and penciclovir after two decades of antiviral therapy. Clin Microbiol Rev. 2003;16:114-28.

52. van Velzen M, van de Vijver DA, van Loenen FB, Osterhaus AD, Remeijer L, Verjans GM. Acyclovir prophylaxis predisposes to antiviral resistant recurrent herpetic keratitis. J Infect Dis. 2013.

53. Subhan S, Jose RJ, Duggirala A, et al. Diagnosis of herpes simplex virus-1 keratitis: comparison of Giemsa stain, immunofluorescence assay and polymerase chain reaction. Curr Eye Res. 2004;29:209-13.

54. Farhatullah S, Kaza S, Athmanathan S, et al. Diagnosis of herpes simplex virus-1 keratitis using Giemsa stain, immunofluorescence assay, and polymerase chain reaction assay on corneal scrapings. Br J Ophthalmol. 2004;88:142-4.

55. Mocan MC, Irkec M, Mikropoulos DG, et al. In vivo confocal microscopic evaluation of the inflammatory response in non-epithelial herpes simplex keratitis. Curr Eye Res. 2012;37:1099-106.

56. Gunduz K, Ozdemir O. Topical cyclosporin as an adjunct to topical acyclovir treatment in herpetic stromal keratitis. Ophthalmic Res. 1997;29:405-8.

57. Sheppard JD, Wertheimer ML, Scoper SV. Modalities to decrease stromal herpes simplex keratitis reactivation rates. Arch Ophthalmol. 2009;127:852-6.

58. Bettahi I, Nesburn AB, Yoon S, et al. Protective immunity against ocular herpes infection and disease induced by highly immunogenic self-adjuvanting glycoprotein D lipopeptide vaccines. Invest Ophthalmol Vis Sci. 2007;48:4643-53. 